\title{
Association between lung function and future risks of diabetes, asthma, myocardial infarction, hypertension and all-cause mortality
}

\author{
Chintal H. Shah $\mathbb{1}^{1}$, Robert M. Reed $\mathbb{B}^{2}$, Yulan Liang ${ }^{3}$ and Zafar Zafari ${ }^{1}$
}

${ }^{1}$ Dept of Pharmaceutical Health Services Research, University of Maryland School of Pharmacy, Baltimore, MD, USA. ${ }^{2}$ Division of Pulmonary and Critical Care Medicine, University of Maryland School of Medicine, Baltimore, MD, USA. ${ }^{3}$ Dept of Family and Community Health, University of Maryland School of Nursing, Baltimore, MD, USA.

Corresponding author: Zafar Zafari (zzafari@rx.umaryland.edu)

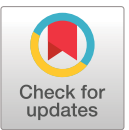

Copyright (The authors 2021

This version is distributed under the terms of the Creative Commons Attribution NonCommercial Licence 4.0. For commercial reproduction rights and permissions contact permissions@ersnet.org

This article has supplementary material available from openres.ersjournals.com

Received: 12 March 2021 Accepted: 4 July 2021

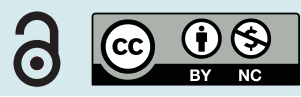

Shareable abstract (@ERSpublications)

These data suggest that lower $\mathrm{FEV}_{1} \%$ predicted values at baseline are significantly associated with a higher future risk of diabetes, asthma and all-cause mortality https://bit.ly/3hVinnB

Cite this article as: Shah $\mathrm{CH}$, Reed RM, Liang Y, et al. Association between lung function and future risks of diabetes, asthma, myocardial infarction, hypertension and all-cause mortality. ERJ Open Res 2021; 7: 00178-2021 [DOI: 10.1183/23120541.00178-2021].

Abstract

Background While forced expiratory volume in $1 \mathrm{~s}\left(\mathrm{FEV}_{1}\right)$ is a hallmark of disease progression in chronic obstructive lung diseases, little is known about the relationship between baseline $\mathrm{FEV}_{1}$ and future risks of other medical conditions.

Objective The aim of this study was to investigate the association between baseline $\mathrm{FEV}_{1}$ and future risks of diabetes, asthma, myocardial infarction, hypertension and all-cause mortality.

Methods We used data from the National Health and Nutrition Examination Survey and its Epidemiological Follow-Up Study. Our data provided longitudinal follow-up of the original cohort for up to 12 years. We used two competing risks approaches, the cause-specific hazard model and the Fine-Gray sub-distribution hazard model, to measure the associations between baseline $\mathrm{FEV}_{1}$ and future risks of the outcomes of interest. All models were adjusted for major confounding factors.

Results The final sample included 3020 participants (mean \pm sD baseline age $44.64 \pm 13.44$ years). In the cause-specific hazard model, for every per cent increase in the baseline per cent predicted $\mathrm{FEV}_{1}$, the hazard of the event reduced by 2.5\% (HR 0.975; 95\% CI 0.958-0.994) for diabetes, 4.3\% (HR 0.957; 95\% CI 0.932-0.983) for asthma and 1.8\% (HR 0.982; 95\% CI 0.971-0.992) for all-cause mortality. There was no statistically significant association between baseline per cent predicted $\mathrm{FEV}_{1}$ and future risks of myocardial infarction (HR 0.987; 95\% CI 0.970-1.004) and hypertension (HR 0.998; 95\% CI 0.9921.005). Consistent results were observed for the Fine-Gray sub-distribution hazard model.

Conclusion Our data suggest that lower per cent predicted $\mathrm{FEV}_{1}$ values at baseline were significantly associated with higher future risks of diabetes, asthma and all-cause mortality.

\section{Introduction}

Pulmonary tests are the mainstay for identification and management of respiratory diseases [1]. Currently, spirometry is the standard test for measuring lung function [2] by quantifying the rate and volume of air flow expired [3]. The forced expiratory volume in $1 \mathrm{~s}\left(\mathrm{FEV}_{1}\right)$ is a key measure of spirometry [1] and represents the maximal volume of air exhaled in the first second of a forced expiration after a position of full inspiration [4].

While $\mathrm{FEV}_{1}$ is a hallmark of disease progression among COPD patients, evidence suggests a linkage between $\mathrm{FEV}_{1}$ and multiple other chronic conditions and mortality [5]. For instance, previous ad hoc studies have found that lung function is inversely associated with the incidence of diabetes [6, 7], heart disease [8, 9], asthma [10], increased blood pressure [11, 12] and mortality [13-17]. Nevertheless, the 
association between $\mathrm{FEV}_{1}$ and future events and the competing nature of such events in a unified model has been understudied.

In this study, we aim to examine possible relationships between baseline $\mathrm{FEV}_{1}$ and future incidence of four major medical conditions including diabetes, asthma, myocardial infarction and hypertension, as well as all-cause mortality using longitudinal observational data. For our analyses, we employ two competing risks frameworks, the cause-specific and the Fine-Gray sub-distribution hazard models.

Methods

Data

We analysed data from the publicly available National Health and Nutrition Examination Survey (NHANES I) and its corresponding Epidemiological Follow-Up Study (NHEFS) [18]. NHANES I included baseline information for a representative sample of the US population aged 25-74 years and was conducted between 1971 and 1975 [18]. NHEFS was a longitudinal follow-up on NHANES I initiated by the National Centre for Health Statistics and the National Institute on Aging in collaboration with other public health service agencies [19]. The NHEFS longitudinal follow-up was designed to investigate the relationship between clinical, behavioural and nutritional factors examined in NHANES I and their long-term effects on morbidity, mortality and hospital utilisation. NHANES I's participants aged 25-74 years, with a complete medical examination in NHANES I, underwent further follow-up investigation on their morbidity, mortality, hospital utilisation, changes in risk factors, and functional limitations for the period between their baseline interview and the first follow-up in 1982-1984 as a part of the NHEFS [19]. The data files utilised are outlined in figure 1.

\section{Primary outcomes and independent variables}

We modelled five medical conditions as our outcomes of interest. The outcomes were diabetes, asthma, myocardial infarction/heart attack, hypertension and all-cause mortality. We chose these medical conditions

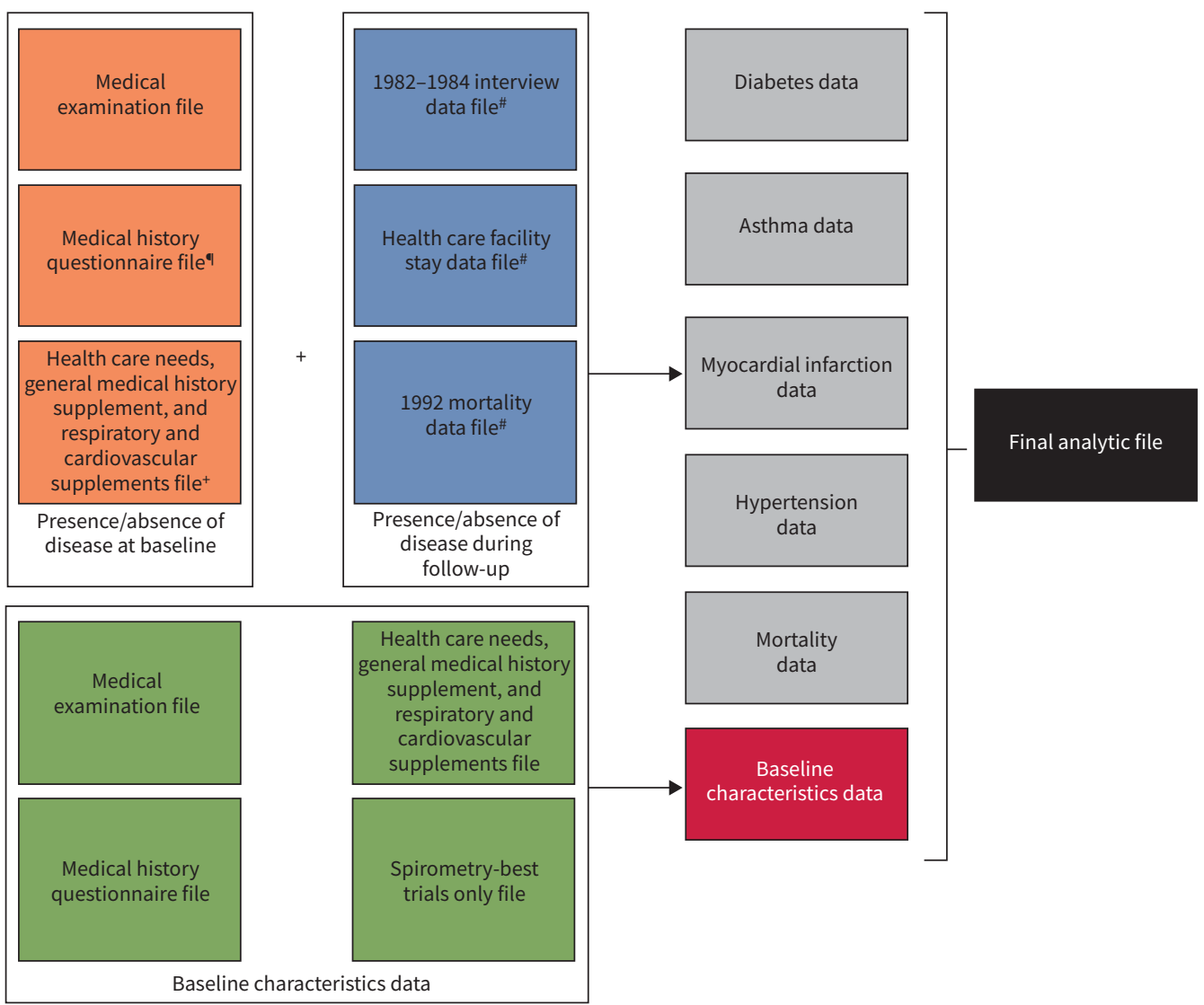

FIGURE 1 Overview of data files used to create the final analytical file. "* data obtained from National Health and Nutrition Examination Survey (NHANES I) Epidemiologic Follow-up Study (NHEFS). ^: used for information on baseline asthma, myocardial infarction and hypertension. ${ }^{+}$: used for information on baseline diabetes. 
for two reasons: first, because they are major events associated with lung function with evidence from prior studies; and second, because we had complete, consistent follow-up data available for these events in NHEFS. The competing events were defined as incident cases over the follow-up period for a maximum of 12 years from the baseline examination until 1982.

Our main independent variables were per cent predicted $\mathrm{FEV}_{1}$ (measured by \%) and absolute $\mathrm{FEV}_{1}$ (measured by litre, L). The spirometry was carried out using an electronic spirometer (Model "800" electric spirometer manufactured by Ohio Medical Instruments Corporation, Cincinnati, OH, USA) [20]. Further information is available elsewhere [20]. The per cent predicted $\mathrm{FEV}_{1}$ was calculated by using the equation provided by HANKINSON et al. [21]. In addition, the presence of COPD at baseline was defined based on the Global Initiative for Chronic Obstructive Lung Disease (GOLD) criterion of having $\mathrm{FEV}_{1}$ / forced vital capacity (FVC) ratio $<0.7$ [5]. Therefore, we utilised the $\mathrm{FEV}_{1} / \mathrm{FVC}$ ratio threshold as a covariate in the model.

In line with previous literature [6], we adjusted for other major covariates in the model including age, body mass index (BMI) $\left(\mathrm{kg} / \mathrm{m}^{2}\right)$, sex, race (black or white), education, smoking status, physical inactivity, marital status, alcohol consumption levels, $\mathrm{FEV}_{1} / \mathrm{FVC}$ ratio threshold and Charlson's Comorbidity Index score [22, 23]. In addition, to account for the possible "U-shape" relationship of BMI with mortality, we included BMI-squared as a covariate in the model $[24,25]$. All covariates were measured at the baseline period.

\section{Defining incident cases of diabetes, asthma, myocardial infarction, hypertension and all-cause mortality}

We used the 1982-1984 NHEFS follow-up file to determine occurrence, and the time of occurrence, of any of the conditions of interest during the follow-up period. In addition to self-reported physician diagnosis, for validation, we used the NHEFS healthcare services utilisation files to determine if individuals had healthcare facility usage for any of the events of interest in the follow-up period. These files were created based on reports of the respondents and subsequent review of the subjects' medical record that was carried out by contacting the healthcare facility.

To capture the association of baseline $\mathrm{FEV}_{1}$ with future incidence of our main conditions/events, we created a cohort of only susceptible (“disease free”) individuals at baseline. That is, we included only individuals who were at risk of developing our main events during the follow-up period, and those with a diagnosis before or during the baseline period were excluded. The combination of the medical examination file (using 3-digit International Classification of Diseases (Ninth Revision), Clinical Modification (ICD9 $\mathrm{CM})$ ), the healthcare needs file and the medical history file from NHANES I were used to ascertain whether the condition of interest was a prevalent one (defined as a condition that was present at baseline). In particular, for any event of interest, a respondent was considered to have diagnosis for any of the conditions in question at baseline if he/she:

- had the condition at baseline as determined from the healthcare needs file or the medical history file; or

- had the condition at baseline as determined from the medical examination file; or

- self-reported physician diagnosis of the condition at any time before or during the baseline period. Individuals with uncertain answers such as those who reported "didn't know” about their event or reported "not ascertained" about the onset time of their event during the follow-up period were excluded. The cohort selection process is illustrated in figure 2 .

If a person did not have any events of interest during the follow-up period, he/she was censored in 1982 in our study. In rare situations, if more than one competing event $(=n)$ was experienced by an individual during a specific year, the same time-to-event was applied to those events with each event having a weight of $1 / n$.

\section{Statistical model}

We developed two competing risks models to study the association of baseline $\mathrm{FEV}_{1}$ with future risks of our events of interest. First, we used a cause-specific hazard model that quantifies the instantaneous risk of an event of interest at a given time, given that the individual has survived, and has not yet experienced any of the competing events (including the event of interest), up to that time point [26]. Therefore, in this approach, the risk set at a given time includes individuals who are free from any competing events up to that time point. 


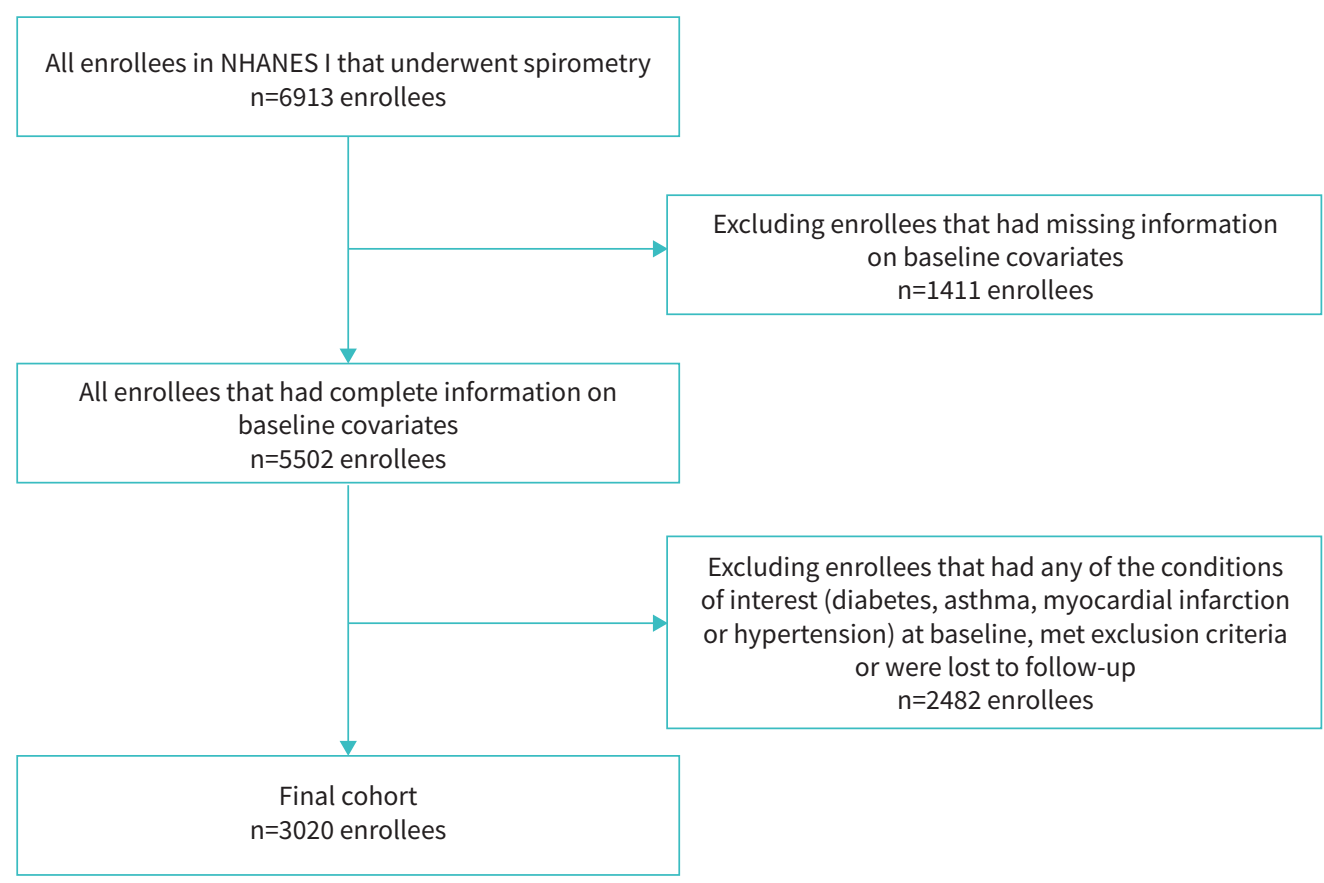

FIGURE 2 Flow diagram illustrating the cohort selection process. NHANES I: National Health and Nutrition Examination Survey.

Second, we used the Fine-Gray sub-distribution hazard model that quantifies the cumulative incidence risk of an event of interest at a given time in the presence of other competing events. This approach models the instantaneous risk of an event at a given time, given that the individual has survived, and has not yet developed the primary event of interest; however, the person was allowed to have developed other competing events besides the primary event up to that time point [26-28]. Therefore, the risk set at time $t$ includes those who are free from the event of interest until time $t$, irrespective of whether or not they have already developed a competing event earlier [26].

All statistical analyses were performed using SAS (version 9.4 SAS Institute Inc., Cary, NC, USA).

Results

After excluding individuals with missing information during the follow-up visits (5.8\%), the final study sample consisted of 3020 individuals. When we compared the baseline per cent predicted $\mathrm{FEV}_{1}$ for the individuals with missing information during the follow-up visits against the final sample, the difference was not statistically significant. The final sample had a mean \pm sD age of $44.64 \pm 13.44$ years at baseline, was predominantly female (54\%) and white (93\%). The mean \pm sD BMI was $24.68 \pm 4.32 \mathrm{~kg} \cdot \mathrm{m}^{-2}$, and $85 \%$ of the sample had a minimum of high school education at baseline. Approximately $15 \%$ of the sample had $\mathrm{FEV}_{1} / \mathrm{FVC}<0.7$, the mean \pm sD $\mathrm{FEV}_{1}$ value was $3.02 \pm 0.84 \mathrm{~L}$ and the mean \pm sD per cent predicted $\mathrm{FEV}_{1}$ was $91.04 \pm 16.26 \%$ at baseline. Also, $60 \%$ of the sample were ever-smokers at baseline. The details of other sample characteristics are displayed in table 1.

\section{Cause-specific hazard model}

Regression model with per cent predicted FEV 1

In the adjusted model, every per cent increase in the baseline per cent predicted $\mathrm{FEV}_{1}$ was associated with a reduced future risk of diabetes of $2.5 \%$ (hazard ratio (HR) 0.975 ; 95\% CI 0.958-0.994), reduced risk of asthma of $4.3 \%$ (HR 0.957 ; 95\% CI $0.932-0.983$ ) and reduced risk of all-cause mortality of $1.8 \%$ (HR 0.982; 95\% CI 0.971-0.992). The association of baseline per cent predicted $\mathrm{FEV}_{1}$ and future risks of myocardial infarction (HR 0.987; 95\% CI 0.970-1.004) and hypertension (HR 0.998; 95\% CI 0.9921.005) were not statistically significant at a two-tailed p-value of 0.05 (table 2). The effects of the other covariates on future incidence of the events of interest are presented in the supplementary Appendix A. 
TABLE 1 Descriptive characteristics of the study cohort at baseline (sample size=3020)

\begin{tabular}{|c|c|c|c|}
\hline Covariate & Entire cohort & Female & Male \\
\hline Age years & $44.64 \pm 13.44(25-74)$ & $43.85 \pm 13.04(25-74)$ & $45.57 \pm 13.85(25-74)$ \\
\hline Body mass index $\mathrm{kg} \cdot \mathrm{m}^{-2}$ & $24.68 \pm 4.32(12.59-53.58)$ & $24.15 \pm 4.71(12.58-53.58)$ & $25.31 \pm 3.71(14.42-46.95)$ \\
\hline Charlson's Comorbidity Index Score & $0.06 \pm 0.32(0-7.00)$ & $0.05 \pm 0.29(0-6.00)$ & $0.08 \pm 0.35(0-7.00)$ \\
\hline $\mathrm{FEV}_{1}$ & $3.02 \pm 0.84(0.36-6.16)$ & $2.64 \pm 0.58(0.68-4.97)$ & $3.47 \pm 0.89(0.36-6.16)$ \\
\hline$\%$ predicted $\mathrm{FEV}_{1}$ & $91.04 \pm 16.26(12.06-195.71)$ & $92.24 \pm 15.72(31.22-195.71)$ & $89.62 \pm 16.78(12.06-184.88)$ \\
\hline Baseline FEV $_{1} /$ FVC $<0.7$ & $431(14.27)$ & $149(9.07)$ & $282(20.46)$ \\
\hline White race & $2803(92.81)$ & $1526(92.94)$ & $1277(92.67)$ \\
\hline At least high school education & $2569(85.07)$ & $1451(88.37)$ & $1118(81.13)$ \\
\hline Ever-smoker" & $1814(60.07)$ & $815(49.63)$ & $999(72.50)$ \\
\hline Physically inactive $^{+}$ & $232(7.68)$ & $117(7.13)$ & $115(8.35)$ \\
\hline Currently married & $2492(82.52)$ & $1308(79.66)$ & $1184(85.92)$ \\
\hline \multicolumn{4}{|l|}{ Drinking frequency (alcohol) } \\
\hline Four or less alcoholic drinks in month & $2099(69.50)$ & $1298(79.05)$ & $801(58.13)$ \\
\hline More than four alcoholic drinks in month & $921(30.50)$ & $344(20.95)$ & $577(41.87)$ \\
\hline Total & $3020(100)$ & $1642(100)$ & $1378(100)$ \\
\hline
\end{tabular}

Data are presented as mean \pm SD (range) or frequency (\%), with percentages presented as a percentage of the column total. FEV ${ }_{1}$ : forced expiratory volume in $1 \mathrm{~s}$; FVC: forced vital capacity. "\#: conditions (weight) included in the calculation of the Charlson's Comorbidity Index at baseline: congestive heart failure (1), peripheral vascular disease (1), cerebrovascular disease (1), dementia (1), connective tissue disease-rheumatic disease (1), peptic ulcer disease (1), mild liver disease (1), paraplegia and hemiplegia (2), renal disease (2), cancer (2), moderate or severe liver disease (3), metastatic carcinoma (6) and HIV/AIDS (6). ": ever-smokers are defined as individuals that were current smokers or former smokers at the time of the baseline interview. ${ }^{+}$: individuals are defined as physically inactive if they responded, "Quite inactive" to the question "In your usual day aside from recreation, how active are you?" at the baseline interview.

Regression model with absolute FEV

Every litre increase in the baseline absolute $\mathrm{FEV}_{1}$ was associated with a reduced instantaneous future risk of diabetes of 55\% (HR 0.452; 95\% CI 0.270-0.755), reduced risk of asthma of 79\% (HR 0.213; 95\% CI 0.096-0.474), reduced risk of myocardial infarction of 40\% (HR 0.599; 95\% CI 0.365-0.984) and reduced risk of all-cause mortality of $41 \%$ (HR 0.588 ; 95\% CI $0.426-0.810$ ). The association between the baseline $\mathrm{FEV}_{1}$ and future risk of hypertension was not statistically significant (HR 0.872; 95\% CI 0.719-1.057) (table 3). The effects of the other covariates in the regression on future incidence of the events of interest are presented in the supplementary Appendix B.

TABLE 2 Results of the cause-specific and Fine-Gray sub-distribution hazard models representing hazard ratio for the incidence of the event of interest during the follow-up period for every $\%$ increase in $\%$ predicted forced expiratory volume in $1 \mathrm{~s}$ at baseline

\begin{tabular}{|c|c|c|c|}
\hline & Hazard ratio & $95 \%$ hazard ratio confidence limits & $\mathrm{p}$-value \\
\hline \multicolumn{4}{|l|}{ Cause-specific hazard } \\
\hline Diabetes & 0.975 & $0.958-0.994$ & $<0.01$ \\
\hline Asthma & 0.957 & $0.932-0.983$ & $<0.01$ \\
\hline Heart attack/myocardial infarction & 0.987 & $0.970-1.004$ & 0.12 \\
\hline Hypertension & 0.998 & $0.992-1.005$ & 0.66 \\
\hline All-cause mortality & 0.982 & $0.971-0.992$ & $<0.01$ \\
\hline \multicolumn{4}{|l|}{ Sub-distribution hazard } \\
\hline Diabetes & 0.977 & $0.959-0.996$ & 0.02 \\
\hline Asthma & 0.959 & $0.937-0.982$ & $<0.01$ \\
\hline Heart attack/myocardial infarction & 0.989 & $0.976-1.002$ & 0.10 \\
\hline Hypertension & 1.000 & $0.993-1.007$ & 0.99 \\
\hline All-cause mortality & 0.984 & $0.974-0.993$ & $<0.01$ \\
\hline
\end{tabular}


TABLE 3 Results of the cause-specific and Fine-Gray sub-distribution hazard models representing hazard ratio for the incidence of the event of interest during the follow-up period for every litre increase in absolute forced expiratory volume in $1 \mathrm{~s}$ at baseline

Hazard ratio $\quad 95 \%$ hazard ratio confidence limits

p-value

\begin{tabular}{|c|c|c|c|}
\hline \multicolumn{4}{|l|}{ Cause-specific hazard } \\
\hline Diabetes & 0.452 & $0.270-0.755$ & $<0.01$ \\
\hline Asthma & 0.213 & $0.096-0.474$ & $<0.01$ \\
\hline Heart attack/myocardial infarction & 0.599 & $0.365-0.984$ & 0.04 \\
\hline Hypertension & 0.872 & $0.719-1.057$ & 0.16 \\
\hline All-cause mortality & 0.588 & $0.426-0.810$ & $<0.01$ \\
\hline \multicolumn{4}{|l|}{ Sub-distribution hazard } \\
\hline Diabetes & 0.473 & $0.273-0.821$ & $<0.01$ \\
\hline Asthma & 0.235 & $0.119-0.463$ & $<0.01$ \\
\hline Heart attack/myocardial infarction & 0.646 & $0.443-0.942$ & 0.02 \\
\hline Hypertension & 0.912 & $0.756-1.099$ & 0.33 \\
\hline All-cause mortality & 0.626 & $0.464-0.845$ & $<0.01$ \\
\hline
\end{tabular}

\section{Fine-Gray sub-distribution hazard model}

Regression model with per cent predicted FEV

Every per cent increase in the per cent predicted $\mathrm{FEV}_{1}$ at baseline was associated with a $2.3 \%$ decrease in the instantaneous risk of diabetes (HR 0.977; 95\% CI 0.959-0.996), 4.1\% decrease in the risk of asthma (HR 0.959; 95\% CI 0.937-0.982) and 1.6\% decrease in the risk of all-cause mortality (HR 0.984; 95\% CI 0.974-0.993). The associations with myocardial infarction (HR 0.989; 95\% CI 0.976-1.002) and hypertension (HR 1.000; 95\% CI 0.993-1.007) were not statistically significant (table 2 and supplementary Appendix A).

Regression model with absolute FEV

Every litre increase in absolute $\mathrm{FEV}_{1}$ at baseline was associated with a $53 \%$ decrease in the instantaneous risk of diabetes (HR 0.473; 95\% CI 0.273-0.821), 76\% decrease in the risk of asthma (HR 0.235; 95\% CI $0.119-0.463$ ), 35\% decrease in the risk of myocardial infarction (HR 0.646; 95\% CI 0.443-0.942) and $37 \%$ decrease in the risk of all-cause mortality (HR 0.626; 95\% CI 0.464-0.845). The association with hypertension was not statistically significant (HR 0.912; 95\% CI 0.756-1.099) (see table 3 and supplementary Appendix B).

\section{Discussion}

In this study, we examined the possible association of baseline $\mathrm{FEV}_{1}$ and future risks of four major medical conditions, namely diabetes, asthma, myocardial infarction/heart attack and hypertension, and all-cause mortality using data from NHANES I and the Epidemiological Follow-Up Study. Our results indicate that $\mathrm{FEV}_{1}$ may play a key role in the long-term incidence of various medical conditions. We used two competing risks modelling approaches. First, using a cause-specific hazard model, our results showed that every per cent decrease in per cent predicted $\mathrm{FEV}_{1}$ at baseline was statistically significantly associated with a $2.5 \%$ higher risk of diabetes, $4.3 \%$ higher risk of asthma and $1.8 \%$ higher risk of all-cause mortality in the future. Our data did not find statistically significant associations between baseline per cent predicted $\mathrm{FEV}_{1}$ and future risks of myocardial infarction and hypertension at the significance level of 0.05 for the given sample size, although the directions of the point estimates were indicative of a possible association between low baseline $\mathrm{FEV}_{1}$ and future risks of these conditions.

Second, we used a Fine-Gray sub-distribution hazard model. The results of this model were consistent with those of the cause-specific hazard model indicative of statistically significant higher future risks of diabetes, asthma and all-cause mortality associated with lower baseline per cent predicted $\mathrm{FEV}_{1}$ values.

The relationship between the baseline $\mathrm{FEV}_{1}$ and future incidence of diabetes in our study is in the same direction as with the previous studies. An earlier population-based Swedish cohort study found that for every additional litre in absolute $\mathrm{FEV}_{1}$, the odds of diabetes would be reduced by 59\% [7]. Despite a 
different methodology, our estimate was quite similar to the Swedish study (55\%). Another study in the USA found that every litre increase in $\mathrm{FEV}_{1}$ (L) was associated with a hazard ratio of 0.698 (p-value <0.001) for diabetes [6]. In our study the corresponding cause-specific hazard ratio was 0.452 ( $p$-value $<0.01$ ). The relationship between $\mathrm{FEV}_{1}$ and diabetes was of particular interest, especially since we adjusted for BMI, a potential confounder, in the regression.

For the association of lung function and future risk of asthma, an earlier study found that reduced lung function at infancy is linked with a greater risk of development of asthma later in life among children [10]. While our results, in an adult population, point in a similar direction, our estimates cannot be directly compared against those of the previous study; this is because the previous study did not measure lung function by absolute $\mathrm{FEV}_{1}$.

In addition, for all-cause mortality, our results are in concordance with the previous studies [14, 17, 29, 30]. For example, SchünEmann et al. [17] found that the all-cause mortality reduced by 1.5\% (HR 0.985; 95\% CI $0.980-0.990)$ and 1\% (HR 0.990; 95\% CI 0.985-0.995) for every per cent increase in per cent predicted $\mathrm{FEV}_{1}$ among men and women, respectively. In another study, LEIVSETH et al. [29] reported that for every $10 \%$ decrease in per cent predicted $\mathrm{FEV}_{1}$, the adjusted hazard ratio for all-cause mortality was 1.17 (95\% CI 1.09-1.25) in women and 1.23 (95\% CI 1.16-1.30) in men. Similarly, our study found that for every per cent increase in the baseline per cent predicted $\mathrm{FEV}_{1}$, the instantaneous risk of all-cause mortality would reduce by $1.8 \%$ (HR 0.982; 95\% CI 0.971-0.992).

Our study has major strengths. First, we conducted our analyses based on a nationally representative dataset with a long follow-up time to quantify the future incidence of the conditions of interest. Second, we looked at the association of the baseline $\mathrm{FEV}_{1}$ and future risks of multiple conditions in a "unified" competing risks framework. For our analyses, we used two popular, yet statistically distinct, competing risks methodologies: the cause-specific hazard model and the Fine-Gray sub-distribution hazard model. While our study does not provide evidence on the causal relationships between the outcomes of interest modelled here, it provides evidence on the competing risks nature of these outcomes.

Our analyses faced some limitations as well. For instance, similar to many observational studies, the inability to adjust for pack-years among smokers and utilisation of medications by participants in our data may lead to certain biases due to unmeasured confounding. Further, patients with lower $\mathrm{FEV}_{1}$ values may visit their healthcare provider more often than their otherwise healthier counterparts, and this could possibly give them a greater chance of being diagnosed with the conditions of interest. However, for the most part, our results were similar to and consistent with previous studies, including prospective studies. Moreover, given that our study only examined five competing outcomes of interest in a non-institutionalised, relatively healthy US sample, cautions should be exercised in the interpretation of our findings. Also, similar to other observation studies, there could be a possibility of undiagnosed individuals with the diseases of interest being included in our final cohort. Finally, while our long-term follow-up could be viewed as a strength, it could also lead to potential changes in diagnostic criteria and treatment options over time that might impact outcomes [31].

Our findings in its entirety suggest that taking steps that result in the preservation of lung function can be associated with long-term reductions in the risks of the medical conditions modelled in this study. As such, investments in interventions that preserve lung function can be potentially associated with health and economic benefits well beyond those that have been already quantified in COPD and other respiratory diseases.

\section{Conclusion}

In our data, lower per cent predicted $\mathrm{FEV}_{1}$ values at baseline were found to be statistically significantly associated with a higher future incidence of diabetes, asthma and all-cause mortality. Our analyses for absolute $\mathrm{FEV}_{1}$ yielded similar results for the future incidence of myocardial infarction. Future studies should further investigate these associations and examine other conditions in different patient populations.

Provenance: Submitted article, peer reviewed.

Author contributions: Z. Zafari developed the study idea and designed the methodological approach; C.H. Shah analysed data and wrote the first draft; R.M. Reed provided clinical insight; Y. Liang provided statistical input; and all authors contributed to the writing. 
Conflict of interest: C.H. Shah has nothing to disclose. R.M. Reed has nothing to disclose. Y. Liang has nothing to disclose. Z. Zafari reports that his research has been funded by the American Lung Association, the Institute for Clinical and Translation Research, the US Food and Drug Administration, the Maryland Dept of Transportation and GSK Pharmaceutical.

Support statement: This study was funded by the American Lung Association and the Institute for Clinical and Translational Research. The funders had no role in study design, data collection and analysis, decision to publish, or preparation of the manuscript. Funding information for this article has been deposited with the Crossref Funder Registry.

References

1 Ranu H, Wilde M, Madden B. Pulmonary function tests. Ulster Med J 2011; 80: 84-90.

2 American Lung Association. Lung Function Tests. www.lung.org/lung-health-and-diseases/lung-proceduresand-tests/lung-function-tests.html Date last accessed: 21 February 2020.

3 Moore VC. Spirometry: step by step. Breathe 2012; 8: 232-240.

4 Miller MR, Hankinson J, Brusasco V, et al. Standardisation of spirometry. Eur Respir J 2005; 26: 319-338.

5 Vogelmeier CF, Criner GJ, Martinez FJ, et al. Global strategy for the diagnosis, management, and prevention of chronic obstructive lung disease 2017 Report. GOLD Executive Summary. Am J Respir Crit Care Med 2017; 195: 557-582.

6 Ford ES, Mannino DM. Prospective association between lung function and the incidence of diabetes: findings from the National Health and Nutrition Examination Survey Epidemiologic Follow-Up Study. Diabetes Care 2004; 27: 2966-2970.

7 Engström G, Janzon L. Risk of developing diabetes is inversely related to lung function: a population-based cohort study. Diabet Med 2002; 19: 167-170.

8 Schroeder EB, Welch VL, Couper D, et al. Lung function and incident coronary heart disease: the Atherosclerosis Risk in Communities Study. Am J Epidemiol 2003; 158: 1171-1181.

9 Engström G, Hedblad B, Nilsson P, et al. Lung function, insulin resistance and incidence of cardiovascular disease: a longitudinal cohort study. J Intern Med 2003; 253: 574-581.

10 Håland G, Carlsen KCL, Sandvik L, et al. Reduced lung function at birth and the risk of asthma at 10 years of age. N Engl J Med 2006; 355: 1682-1689.

11 Engström G, Wollmer P, Valind S, et al. Blood pressure increase between 55 and 68 years of age is inversely related to lung function: longitudinal results from the cohort study 'Men born in 1914'. J Hypertens 2001; 19: 1203-1208.

12 Sparrow D, Weiss ST, Vokonas PS, et al. Forced vital capacity and the risk of hypertension. The Normative Aging Study. Am J Epidemiol 1988; 127: 734-741.

13 Higgins MW, Keller JB. Predictors of mortality in the adult population of Tecumseh. Arch Environ Health 1970; 21: 418-424.

14 Hole DJ, Watt GC, Davey-Smith G, et al. Impaired lung function and mortality risk in men and women: findings from the Renfrew and Paisley prospective population study. BMJ 1996; 313: 711-716.

15 Miller MR, Pedersen OF, Lange $\mathrm{P}$, et al. Improved survival prediction from lung function data in a large population sample. Respir Med 2009; 103: 442-448.

16 Sabia S, Shipley M, Elbaz A, et al. Why does lung function predict mortality? Results from the Whitehall II Cohort Study. Am J Epidemiol 2010; 172: 1415-1423.

17 Schünemann HJ, Dorn J, Grant BJ, et al. Pulmonary function is a long-term predictor of mortality in the general population: 29-year follow-up of the Buffalo Health Study. Chest 2000; 118: 656-664.

18 CDC. NHANES I (1971-1974). wwwn.cdc.gov/nchs/nhanes/nhanes1/Default.aspx Date last accessed: 9 February 2020.

19 CDC. NHANES I - Epidemiologic Followup Study (NHEFS). wwwn.cdc.gov/nchs/nhanes/nhefs/default.aspx/ Date last accessed: 9 February 2020.

20 U.S. Department of Health and Human Services, Public Health Services, National Center for Health Statistics. Public Use Data Tape Documentation: Spirometry-Best Trials Only Tape number 4250. wwwn.cdc.gov/nchs/ data/nhanes1/4250.pdf Date last accessed: 10 March 2021.

21 Hankinson JL, Odencrantz JR, Fedan KB. Spirometric reference values from a sample of the general U.S. population. Am J Respir Crit Care Med. 1999; 159: 179-187.

22 Charlson ME, Pompei P, Ales KL, et al. A new method of classifying prognostic comorbidity in longitudinal studies: development and validation. J Chronic Dis 1987; 40: 373-383.

23 Deyo RA, Diehr P, Patrick DL. Reproducibility and responsiveness of health status measures statistics and strategies for evaluation. Control Clin Trials 1991; 12: S142-S158.

24 Spelta F, Fratta Pasini AM, Cazzoletti L, et al. Body weight and mortality in COPD: focus on the obesity paradox. Eat Weight Disord 2018; 23: 15-22. 
25 Abston E, Comellas A, Reed RM, et al. Higher BMI is associated with higher expiratory airflow normalised for lung volume (FEF25-75/FVC) in COPD. BMJ Open Respir Res 2017; 4: e000231.

26 Austin PC, Lee DS, Fine JP. Introduction to the analysis of survival data in the presence of competing risks. Circulation 2016; 133: 601-609.

27 Lau B, Cole SR, Gange SJ. Competing risk regression models for epidemiologic data. Am J Epidemiol 2009; 170: 244-256.

28 Fine JP, Gray RJ. A proportional Hazards model for the subdistribution of a competing risk. J Am Stat Assoc 1999; 94: 496-509.

29 Leivseth L, Nilsen TIL, Mai X-M, et al. Lung function and respiratory symptoms in association with mortality: The HUNT Study. COPD 2014; 11: 59-80.

30 Ryan G, Knuiman MW, Divitini ML, et al. Decline in lung function and mortality: the Busselton Health Study. J Epidemiol Community Health 1999; 53: 230-234.

31 Bergsten TM, Nicholson A, Donnino R, et al. Predicting adults likely to develop heart failure using readily available clinical information: an analysis of heart failure incidence using the NHEFS. Prev Med 2020; 130: 105878. 